\title{
THE POISSON-VORONOI TESSELLATION: RELATIONSHIPS FOR EDGES
}

\author{
L. MUCHE*
}

\begin{abstract}
In a unified approach, this paper presents distributional properties of a Voronoi tessellation generated by a homogeneous Poisson point process in the Euclidean space of arbitrary dimension. Probability density functions and moments are given for characteristics of the 'typical' edge in lower-dimensional section hyperplanes (edge lengths, adjacent angles). We investigate relationships between edges and their neighbours, called Poisson points or centres; namely angular distributions, distances, and positions of neighbours relative to the edge. The approach is analytical, and the results are given partly explicitly and partly as integral expressions, which are suitable for the numerical calculations also presented.
\end{abstract}

Keywords: Probability density function; moment; edge length; adjacent angle; distance; neighbor

2000 Mathematics Subject Classification: Primary 60D05

Secondary 52A22

\section{Introduction}

The Voronoi tessellation is a popular stochastic geometrical model applied in many fields of science and engineering; see Okabe et al. [16]. A commonly used special case is that of complete randomness, in which the generating point pattern is a homogeneous Poisson point process. This so-called Poisson-Voronoi tessellation has been studied by many researchers, both analytically and by means of simulation.

Probability density functions of edge lengths of a tessellation generated by a Poisson point process in the two- and three-dimensional Euclidean spaces $\left(\mathbb{R}^{2}\right.$ and $\left.\mathbb{R}^{3}\right)$ were given by Brakke [1], [2]. Møller [11], [12] gave a formula for means of edge lengths in $\mathbb{R}^{d}$, and corresponding $s$-dimensional sections $(1 \leq s \leq d)$, and discussed a method of calculating probability density functions in general. Muche and Stoyan [15] determined the probability density functions of chord lengths generated by linear sections $(s=1)$ through the PoissonVoronoi tessellation in $\mathbb{R}^{d}, 2 \leq d \leq 7$.

Muche [13] provided a method of calculating the probability density functions of edge lengths and gave results in dimension $d, 2 \leq d \leq 5$, with $s=d$ and $s=2$ (planar sections). Muche [14] summarized the case $d=3$ with formulae valid for $1 \leq s \leq 3$, while Schlather [18] gave a method that allows the determination of the probability density functions of edge lengths in arbitrary dimension $d$ and for arbitrary $s$-dimensional section, $1 \leq s \leq d$.

Other characteristics of interest are the distances of an edge to its neighbours (namely centres, generating points, seeds, or atoms - here considered members of the Poisson point process). The neighbourhood of each edge of the Poisson-Voronoi tessellation in $\mathbb{R}^{d}$ is completely characterized by $d+2$ points of the generating Poisson point process: each edge endpoint

Received 21 July 2004; revision received 5 December 2004.

* Postal address: Balthasar-Rössler-Strasse 59, D-09599 Freiberg (Sachs), Germany.

Email address: lutz.muche@gmx.de 
(vertex) is surrounded by $d+1$ Poisson points, which are located on the boundary of a sphere centred at the endpoint. Consequently, $d$ of these Poisson points, called neighbours of the edge, belong to both vertices and lie in a $(d-1)$-dimensional hyperplane perpendicular to the edge, all at the same distance from the edge. (Analogously, an edge in a section of dimension $s, 1 \leq s \leq d$, has $s$ neighbours in a perpendicular $(d-1)$-dimensional hyperplane and one further Poisson point on either side of it.) In the planar case $d=s=2$, there are two neighbours symmetric to the edge. Their distance was studied by Collins [3], [4] and Brakke [1]; Collins also discussed an extension to the spatial case. Furthermore, distributions of the neighbour-vertex-edge angle were studied in [3], [4], and [9]. Probability density functions of vertex-neighbour distances and the angles at vertices were investigated by Miles [10] in the case $d=s=2$. Distributional properties of the neighbourhood around a vertex of the PoissonVoronoi tessellation in $\mathbb{R}^{d}$ were also studied there. These basic formulae are fundamental to the investigation of many other geometrical characteristics of the Poisson-Voronoi tessellation.

This paper takes up methods given in [5], [8], and [13]. In a unified approach, we find general results valid for arbitrary dimension $d \geq 2$ and all corresponding $s$-dimensional sections, $1 \leq s \leq d$. In contrast to earlier results, the formulae given here allow numerical calculations. The method employs a basic identity concerning the distributional properties in the neighbourhood of the 'typical' vertex investigated in [8]; the word 'typical' is used in the sense of [19, p. 117]. Another foundation is the fact that the distribution of the typical edge coincides with the distribution of a randomly chosen edge emanating from the typical vertex; see [8]. Finally, we also exploit the symmetry of distributional properties with respect to both endpoints of the typical edge.

The paper presents probability density functions and moments of

- the length of the typical edge of the Poisson-Voronoi tessellation,

- the angles at the endpoints of the typical edge spanned by a neighbour,

- the angle at a neighbour spanned by the endpoints of the typical edge,

- the distance from a neighbour to the typical edge, and

- the position of a neighbour with respect to the typical edge.

In addition to the classical applications of Voronoi tessellations in agriculture and forestry, astrophysics, cell biology, crystallography, geography, metallography, zoology, and ecology, there are two very recently developed fields, namely telecommunications (see [6]) and molecular physics.

Edges of the Voronoi tessellation play an important role in modern investigations of macromolecular structures; see [7], [17], and references therein. In this context, the separation of neighbouring atoms into pairs of direct and indirect neighbours is important. Two neighbouring atoms are direct neighbours if the straight line (Voronoi edge) between them intersects the corresponding joint Voronoi face (facet); otherwise they are indirect neighbours. The understanding of many phenomena in real structures requires knowledge of properties of Voronoi edges.

The paper is structured as follows. In Section 2, we describe our method and, in Section 3, we present the main results. Further results obtained by application of the method are given in Section 4. In Section 5, we discuss numerical calculations in some special cases and present tables and figures with the results. Finally, the proofs of our results are given in Section 6. 


\section{Fundamentals}

Let $\mathfrak{V}$ be the Voronoi tessellation generated by a stationary and isotropic Poisson point process $\Phi$ of intensity $\lambda$ in the $d$-dimensional Euclidean space $\mathbb{R}^{d}$. The cells of $\mathfrak{V}$ are $d$-dimensional bounded open convex polytopes ( $d$-facets). Their boundaries are pieces of hyperplanes of dimension smaller than $d$, called $(d-1)-,(d-2)-, \ldots, 2-, 1-, 0$-facets. We call 1 -facets edges. Each boundary point can be assigned to one $k$-facet $(0 \leq k<d)$ uniquely. Each $k$-facet is characterized by $d-k+1$ Poisson points, called neighbours, which are all within the same distance from the facet, with no Poisson point closer.

Let $S$ denote an $s$-dimensional section hyperplane through $\mathfrak{V}$, with $s=1,2, \ldots, s \leq d$. Without loss of generality, $S$ can be considered to be the hyperplane spanned by the $x_{1-}, x_{2^{-}}, \ldots, x_{S^{-}}$axes of a Cartesian coordinate system. The intersection $\mathfrak{V}_{s}=\mathfrak{V} \cap S$ is a stationary and isotropic tessellation of dimension $s$. The edges of $\mathfrak{V}_{s}$ are intersections between $(d-s+1)$-facets and the intersection hyperplane $S$, and the vertices of $\mathfrak{V}_{s}$ are intersections of $(d-s)$-facets. Note that the occurrence of intersection points is weighted by the $(d-s)$-volume of the corresponding $(d-s)$-facet. (The concept of $k$-volume is used in the sense of $k$-dimensional Lebesgue measure.) A vertex in $\mathfrak{V}_{s}$ has $s+1$ neighbours, meaning that $s+1$ points of the generating point process $\Phi$ have the same distance to it and there are no neighbours closer. Analogously, an edge in $S$ has $s$ neighbours located on a $(d-1)$-dimensional hyperplane perpendicular to it. We denote the typical vertex of $\mathfrak{V}_{s}$ by $o$ and one of its associated edges by $E_{d, s}^{0}$. Without loss of generality, we denote its neighbours (belonging to the generating point process) by $z_{0}, \ldots, z_{s-1}$, and denote the additional neighbour of $o$ by $z_{s}$. There are thus $s+1$ nearest neighbours having the same distance $\Delta_{d, s}$ to $o$. The distributional properties with respect to the typical vertex were investigated in [8], which is based on the theory of Palm distributions. The basic property given there in Lemma 1 and Lemma $1^{\prime}$, shown for $s=2$ and $s=d$ in detail, is valid for all the other cases $1 \leq s \leq d$, by analogy; see [5]. The Palm version of the point process of vertices of the Poisson-Voronoi tessellation is characterized by a sphere $b\left(o, \Delta_{d, s}\right)$ containing no neighbours in its interior, but $s+1$ neighbours on its boundary. (Here $b(x, y)$ stands for the compact closed $d$-dimensional sphere of radius $y$ centred at $x$, whereas $\partial b(x, y)$ denotes its boundary. $)$ The point pattern in the space outside $b\left(o, \Delta_{d, s}\right)$ can be handled as a Poisson point process.

The typical vertex $o$ of $\mathfrak{V}_{s}$ is the joint endpoint of $s+1$ edges in $\mathfrak{V}_{s}$. Consider one of these $s+1$ edges, say $E_{d, s}^{0}$, chosen at random and each with the same probability. Let $E_{d, s}^{0}$ have second endpoint $\underline{l}$ (with coordinate $l$ on the positive $x_{1}$-axis). Then, the following relationships must hold:

$$
\begin{gathered}
\Phi\left(b^{\text {int }}\left(o,\left\|z_{0}\right\|\right) \cup b^{\text {int }}\left(\underline{l},\left\|z_{0}-\underline{l}\right\|\right)\right)=0, \\
\Phi\left(\partial b\left(o,\left\|z_{0}\right\|\right) \cap \partial b\left(\underline{l},\left\|z_{0}-\underline{l}\right\|\right)\right)=s, \\
\Phi\left(\partial b\left(o,\left\|z_{0}\right\|\right) \backslash b\left(\underline{l},\left\|z_{0}-\underline{l}\right\|\right)\right)=1, \\
\Phi\left(\partial b\left(\underline{l},\left\|z_{0}-\underline{l}\right\|\right) \backslash b\left(o,\left\|z_{0}\right\|\right)\right)=1,
\end{gathered}
$$

where $\|\cdot\|$ denotes the Euclidean norm in $\mathbb{R}^{d}$. In other words, the interior of the union of the two spheres around the endpoints of $E_{d, s}^{0}$ must be empty. The intersection of their boundaries contains $s$ Poisson points (neighbours of $E_{d, s}^{0}$ ), and on either side of them there is a further Poisson point on the rest of the boundary. These geometrical relationships allow 
the determination of the probability density function $f_{L_{d, s}^{0}}$ of the length $L_{d, s}^{0}$ of the edge $E_{d, s}^{0}$ emanating from the typical vertex. The corresponding result of [13] is

$$
f_{L_{d, s}^{0}}(l)=\lambda \int_{0}^{\infty} \int_{0}^{\pi} f_{\Delta_{d, s}}(\delta) f_{B_{d, s}}(\beta) \frac{\mathrm{d} \nu_{d}^{0}(l, \delta, \beta)}{\mathrm{d} l} \exp \left(-\lambda v_{d}^{0}(l, \delta, \beta)\right) \mathrm{d} \beta \mathrm{d} \delta,
$$

where the variables $\delta$ and $\beta$ correspond to the distance $\Delta_{d, s}=\left\|z_{0}\right\|$ and the angle $B_{d, s}=\angle\left(z_{0}, o, \underline{l}\right)$, with the probability density functions $f_{\Delta_{d, s}}(\delta)$ and $f_{B_{d, s}}(\beta)$, respectively, and $v_{d}^{0}(l, \delta, \beta)$ denotes the $d$-volume of the union

$$
b\left(o,\left\|z_{0}\right\|\right) \cup b\left(\underline{l},\left\|z_{0}-\underline{l}\right\|\right)=b(o, \delta) \cup b\left(\underline{l}, \sqrt{l^{2}+\delta^{2}-2 l \delta \cos \beta}\right) .
$$

These results can be translated so as to apply to the typical edge $E_{d, s}^{1}$. From [8], $E_{d, s}^{0}$ and $E_{d, s}^{1}$ have identical distributional properties. This means that the distribution function $F_{E_{d, s}^{0}}^{0}$ of any geometrical characteristic of a randomly chosen edge emanating from the typical vertex is identical to the distribution function $F_{E_{d, s}^{1}}$ of the equivalent characteristic of the typical edge $E_{d, s}^{1}$ :

$$
F_{E_{d, s}^{0}} \equiv F_{E_{d, s}^{1}} .
$$

The proof of (2) in [8, Lemma 2 and Lemma $\left.2^{\prime}\right]$, given, in the respective lemmas, for $s=d$ and $s=2$, can be generalized to the other cases analogously.

The probability density function of the length $L_{d, s}^{1}$ of the typical edge $E_{d, s}^{1}$ of $\mathfrak{V}_{s}$ can be given in the form

$$
\begin{gathered}
f_{L_{d, s}^{1}}(l)=\lambda \int_{0}^{\pi} \int_{0}^{\pi-\beta_{1}} f_{\Delta_{d, s}}\left(l \frac{\sin \beta_{2}}{\sin \left(\beta_{1}+\beta_{2}\right)}\right) f_{B_{d, s}}\left(\beta_{1}\right) v_{d}^{\prime}\left(l, \beta_{1}, \beta_{2}\right)\left|\frac{\partial(\beta, \delta)}{\partial\left(\beta_{1}, \beta_{2}\right)}\right| \\
\quad \times \exp \left(-\lambda v_{d}\left(l, \beta_{1}, \beta_{2}\right)\right) \mathrm{d} \beta_{2} \mathrm{~d} \beta_{1},
\end{gathered}
$$

where $\Delta_{d, s}=\left\|z-v_{1}\right\|$ and we have angles $B_{1_{d, s}}=\angle\left(z, v_{1}, v_{2}\right)$ and $B_{2_{d, s}}=\angle\left(z, v_{2}, v_{1}\right)$. Here, $v_{1}$ and $v_{2}$ denote the endpoints of $E_{d, s}^{1}$ and $z$ denotes one of its $s$ neighbours lying in the $(d-1)$-dimensional hyperplane perpendicular to it. Furthermore, $v_{d}\left(l, \beta_{1}, \beta_{2}\right)$ corresponds to the $d$-volume $v_{d}^{0}(l, \delta, \beta)$ of the union of two overlapping spheres, used in (1), and $v_{d}^{\prime}\left(l, \beta_{1}, \beta_{2}\right)$ corresponds to its derivative $\mathrm{d} v_{d}^{0}(l, \delta, \beta) / \mathrm{d} l$. Thus, (1) can be transformed into (3) by the transformation of variables

$$
\beta=\beta_{1}, \quad \delta=l \frac{\sin \beta_{2}}{\sin \left(\beta_{1}+\beta_{2}\right)}, \quad\left|\frac{\partial(\beta, \delta)}{\partial\left(\beta_{1}, \beta_{2}\right)}\right|=l \frac{\sin \beta_{1}}{\sin ^{2}\left(\beta_{1}+\beta_{2}\right)},
$$

and, conversely, by

$$
\beta_{1}=\beta, \quad \beta_{2}=\operatorname{arccot} \frac{l-\delta \cos \beta}{\delta \sin \beta}, \quad\left|\frac{\partial\left(\beta_{1}, \beta_{2}\right)}{\partial(\beta, \delta)}\right|=\frac{l \sin \beta}{l^{2}-2 l \delta \cos \beta+\delta^{2}} .
$$

Henceforth, we denote the typical edge $E_{d, s}^{1}$ of $\mathfrak{V}_{s}$ by $E_{d, s}$ and the random lengths by

$$
L_{d, s}^{0}=L_{d, s}^{1}=L_{d, s} .
$$

The use of both (1) and (3), identity (2), and the symmetry property

$$
f_{L_{d, s}}\left(l, \beta_{1}, \beta_{2}\right)=f_{L_{d, s}}\left(l, \beta_{2}, \beta_{1}\right)
$$

leads to numerous results. We give these in the following section. 


\section{Characteristics of edges}

This section contains the main results of the paper and generalizes and simplifies earlier results. All numbered equations represent new results explicitly describing the distributional properties of Voronoi edges for all dimensions $d$ and $s$, with $2 \leq d$ and $1 \leq s \leq d$.

A vertex in an $s$-dimensional section $\mathfrak{V}_{s}$ has $s+1$ equidistant nearest neighbours. The probability density function of the distance $\Delta_{d, s}$ of the typical vertex $o$ of $\mathfrak{V}_{s}$ from each of its neighbours is

$$
f_{\Delta_{d, s}}(\delta)=\frac{d\left(\lambda \omega_{d}\right)^{s+1-s / d}}{\Gamma(s+1-s / d)} \delta^{(d-1)(s+1)} \exp \left(-\lambda \omega_{d} \delta^{d}\right), \quad 0 \leq \delta<\infty,
$$

where $\omega_{d}=\pi^{1 / 2} / \Gamma\left(1+\frac{1}{2} d\right)$ denotes the $d$-volume of a unit sphere $b(o, 1)$. This formula generalizes earlier results given in [1] and [9] (for $f_{\Delta_{2,2}}(\delta)$ ), [10] and [12] (for $f_{\Delta_{d, d}}(\delta), d \geq 2$ ), [15] (for $f_{\Delta_{d, 1}}(\delta), d \geq 2$ ), and [13] (for $f_{\Delta_{d, 2}}(\delta)$ and $f_{\Delta_{d, d}}(\delta), 2 \leq d \leq 5$ ). Schlather [18] used (6) implicitly.

The corresponding moments are

$$
\mathrm{E} \Delta_{d, s}^{k}=\frac{\Gamma(s+1+(k-s) / d)}{\Gamma(s+1-s / d)}\left(\frac{1}{\lambda \omega_{d}}\right)^{k / d}, \quad k=0,1,2 \ldots
$$

In (1) and (3), expressions are used for the $d$-volume (the $d$-dimensional Lebesgue measure, recall) of two overlapping spheres and its derivative with respect to the midpoint distance. In the following, expressions for $v_{d}\left(l, \beta_{1}, \beta_{2}\right)$ and $v_{d}^{\prime}\left(l, \beta_{1}, \beta_{2}\right)$ are given for arbitrary dimension $d \geq 2$. The union of two overlapping spheres can be thought of as the union of two truncated spheres and, thus, written as

$$
v_{d}\left(l, \beta_{1}, \beta_{2}\right)=\varpi_{d}\left(l \frac{\sin \beta_{2}}{\sin \left(\beta_{1}+\beta_{2}\right)}, \beta_{1}\right)+\varpi_{d}\left(l \frac{\sin \beta_{1}}{\sin \left(\beta_{1}+\beta_{2}\right)}, \beta_{2}\right) .
$$

Here, the $d$-volume of a truncated sphere of radius $r$ and intersection longitude $\beta$ is given by

$$
\varpi_{d}(r, \beta)=r^{d} \omega_{d} \sum_{i=0}^{\lfloor d / 2\rfloor} a_{i}(\beta)
$$

where $\lfloor x\rfloor$ denotes the greatest whole number smaller $x$ (the integer part of $x$ ) and

$$
\begin{aligned}
& a_{0}(\beta)=\left\{\begin{array}{l}
1-\frac{\beta}{\pi}, \quad d \text { even, } \\
\cos ^{2} \frac{1}{2} \beta, \quad d \text { odd, }
\end{array}\right. \\
& a_{i}(\beta)=\left\{\begin{array}{l}
\frac{\cos \beta}{2 \sqrt{\pi}} \frac{\Gamma(i)}{\Gamma\left(i+\frac{1}{2}\right)} \sin ^{2 i-1} \beta, \quad d \text { even, } \\
\frac{\cos \beta}{2 \sqrt{\pi}} \frac{\Gamma\left(i+\frac{1}{2}\right)}{\Gamma(i+1)} \sin ^{2 i} \beta, \quad d \text { odd, }
\end{array} \quad i=1, \ldots,\left\lfloor\frac{1}{2} d\right\rfloor .\right.
\end{aligned}
$$

For $\beta=\pi$, the $d$-volume vanishes and, for $\beta=0$, the $d$-volume is that of a complete sphere:

$$
\varpi_{d}(1, \pi)=0, \quad \varpi_{d}(1,0)=\omega_{d}=\frac{\pi^{d / 2}}{\Gamma\left(\frac{1}{2} d+1\right)} .
$$


The derivative $v_{d}^{\prime}\left(l, \beta_{1}, \beta_{2}\right)$ of $v_{d}\left(l, \beta_{1}, \beta_{2}\right)$ is

$$
v_{d}^{\prime}\left(l, \beta_{1}, \beta_{2}\right)=d \omega_{d}\left(\frac{l \sin \beta_{1}}{\sin \left(\beta_{1}+\beta_{2}\right)}\right)^{d-1} \sum_{i=0}^{\lfloor(d-1) / 2\rfloor} b_{i}\left(\beta_{2}\right),
$$

with

$$
\begin{aligned}
& b_{0}(\beta)= \begin{cases}\frac{(\pi-\beta) \cos \beta+\sin \beta}{\pi}, & d \text { even, } \\
\cos ^{2} \frac{1}{2} \beta, & d \text { odd, }\end{cases} \\
& b_{i}(\beta)= \begin{cases}-\frac{1}{4 \sqrt{\pi}} \frac{\Gamma(i)}{\Gamma\left(i+\frac{3}{2}\right)} \sin ^{2 i+1} \beta, & d \text { even, } \\
-\frac{1}{4 \sqrt{\pi}} \frac{\Gamma\left(i-\frac{1}{2}\right)}{\Gamma(i+1)} \sin ^{2 i} \beta, & d \text { odd, }\end{cases}
\end{aligned}
$$

Consequently, the probability density function of the neighbour-vertex-edge angle of $E_{d, s}$ is

$$
\begin{aligned}
& f_{B_{d, s}}(\beta)=\frac{(d-1) s \Gamma\left(\frac{1}{2}(d+1)\right) \Gamma\left(\frac{1}{2}(d s+d-s)\right)}{\Gamma\left(\frac{1}{2} d\right) \Gamma\left(\frac{1}{2}(d s+d-s+1)\right)} \sin ^{d s-s-1} \beta \sum_{i=0}^{\lfloor(d-1) / 2\rfloor} b_{i}(\beta), \\
& 0 \leq \beta<\pi .
\end{aligned}
$$

This formula is a generalization of results given in [1], [3], [9], and [12] (for $f_{B_{2,2}}(\beta)$ ), [13] (for $f_{B_{d, s}}(\beta)$ with $\left.2 \leq d \leq 5, s=2, d\right)$, and [14] (for $f_{B_{3, s}}(\beta), s=1,2,3$ ). A more complicated formula, equivalent to (13), containing an integral expression is given in [18].

The corresponding moments are

$$
\mathrm{E} B=\frac{1}{2} \pi-\frac{\sqrt{\pi}}{2} \frac{\Gamma\left(\frac{1}{2}(d+1)\right) \Gamma\left(\frac{1}{2}(d s+d-s)\right) \Gamma\left(\frac{1}{2}(d s-s+1)\right)}{\Gamma\left(\frac{1}{2} d\right) \Gamma\left(\frac{1}{2}(d s+d-s+1)\right) \Gamma\left(\frac{1}{2}(d s-s+2)\right)},
$$

$\mathrm{E}(\sin B)^{k}=\frac{(d-1) s \Gamma\left(\frac{1}{2}(d s+d-s)\right) \Gamma\left(\frac{1}{2}(d s+d+k-s+1)\right)}{(d s+k-s) \Gamma\left(\frac{1}{2}(d s+d-s+1)\right) \Gamma\left(\frac{1}{2}(d s+d+k-s)\right)}, \quad k=0,1,2, \ldots$,

$\mathrm{E}(\cos B)^{k}=\frac{\Gamma\left(\frac{1}{2}(d+1)\right) \Gamma\left(\frac{1}{2}(d s+d-s)\right) \Gamma\left(\frac{1}{2}(d s-s+2)\right) \Gamma\left(\frac{1}{2}(k+2)\right)}{\Gamma\left(\frac{1}{2} d\right) \Gamma\left(\frac{1}{2}(d s+d-s+1)\right) \Gamma\left(\frac{1}{2}(d s+k-s+2)\right)}, \quad k$ odd.

The joint probability density function of the length $L$ of the typical edge $E_{d, s}$ of $\mathfrak{V}_{s}$ and the two adjacent angles $B_{1}$ and $B_{2}$ spanned by its neighbours is

$$
\begin{aligned}
f_{\left\{L, B_{1}, B_{2}\right\}_{d, s}\left(l, \beta_{1}, \beta_{2}\right)} & \frac{2 d \pi^{[d(s+2)-s] / 2} \Gamma\left(\frac{1}{2}(d s+d-s+1)\right) \lambda^{s+2-s / d}}{(d-1) s \Gamma\left(\frac{1}{2}(d+1)\right) \Gamma\left(\frac{1}{2}(d s+d-s)\right) \Gamma(s+1-s / d)\left\{\Gamma\left(\frac{1}{2} d+1\right)\right\}^{s+1-s / d}} \\
& \times \frac{l^{d(s+2)-s-1}\left(\sin \beta_{1} \sin \beta_{2}\right)^{d}}{\sin ^{d s+2 d-s}\left(\beta_{1}+\beta_{2}\right)} f_{B_{1}}\left(\beta_{1}\right) f_{B_{2}}\left(\beta_{2}\right) \exp \left(-\lambda v_{d}\left(l, \beta_{1}, \beta_{2}\right)\right)
\end{aligned}
$$


or, equivalently,

$f_{\left\{L, B_{1}, B_{2}\right\}_{d, s}}\left(l, \beta_{1}, \beta_{2}\right)$

$$
\begin{aligned}
= & c_{d, s} \lambda^{s+2-s / d} l^{d(s+2)-s-1} \frac{\left(\sin \beta_{1} \sin \beta_{2}\right)^{d s+d-s-1}}{\sin ^{d s+2 d-s}\left(\beta_{1}+\beta_{2}\right)}\left(\sum_{i=0}^{\lfloor(d-1) / 2\rfloor} b_{i}\left(\beta_{1}\right)\right)\left(\sum_{i=0}^{\lfloor(d-1) / 2\rfloor} b_{i}\left(\beta_{2}\right)\right) \\
& \times \exp \left(-\lambda \frac{\pi^{d / 2} l^{d}}{\Gamma\left(\frac{1}{2} d+1\right)}\left\{\frac{\sin ^{d} \beta_{2}}{\sin ^{d}\left(\beta_{1}+\beta_{2}\right)} \sum_{i=0}^{\lfloor d / 2\rfloor} a_{i}\left(\beta_{1}\right)+\frac{\sin ^{d} \beta_{1}}{\sin ^{d}\left(\beta_{1}+\beta_{2}\right)} \sum_{i=0}^{\lfloor d / 2\rfloor} a_{i}\left(\beta_{2}\right)\right\}\right),
\end{aligned}
$$

with $0 \leq l<\infty, 0 \leq \beta_{1}<\pi, 0 \leq \beta_{2}<\pi-\beta_{1}$, and

$$
c_{d, s}=\frac{d^{4} \pi^{[d(s+2)-s] / 2} \Gamma\left(\frac{1}{2}(d+1)\right) \Gamma\left(\frac{1}{2}(d s+d-s)\right)}{2\left\{\Gamma\left(\frac{1}{2} d+1\right)\right\}^{s+3-s / d} \Gamma(s-s / d) \Gamma\left(\frac{1}{2}(d s+d-s+1)\right)} .
$$

The probability density function of the length of the typical edge $E_{d, s}$ of $\mathfrak{V}_{s}$ is given by

$$
f_{L_{d, s}}(l)=\int_{0}^{\pi} \int_{0}^{\pi-\beta_{1}} f_{\left\{L, B_{1}, B_{2}\right\}_{d, s}}\left(l, \beta_{1}, \beta_{2}\right) \mathrm{d} \beta_{2} \mathrm{~d} \beta_{1} .
$$

The formulae (17), (18), and (19) generalize results given in [1] (for $f_{L_{2,2}}(l)$ ), [2] (for $f_{L_{3,3}}(l)$ ), [12] (for $f_{L_{d, d}}(l), d=2,3$ ), [15] (for $f_{L_{d, 1}}(l), 2 \leq d \leq 7$ ), [13] (for $f_{L_{d, 2}}(l)$ and $f_{L_{d, d}}(l)$, $2 \leq d \leq 5$ ), and [14] (for $f_{L_{3, s}}(l), s=1,2,3$ ). An equivalent general expression is given in [18]. to

The formula for the moments of $L_{d, s}$, which is $\mathrm{E} L_{d, s}^{k}=\int_{0}^{\infty} l^{k} f_{L_{d, s}}(l) \mathrm{d} l$, can be simplified

$$
\begin{aligned}
& \mathrm{E} L_{d, s}^{k}= \frac{c_{d, s} \Gamma(s+2+(k-s) / d)\left\{\Gamma\left(\frac{1}{2} d+1\right)\right\}^{s+2+(k-s) / d}}{d \pi^{[d(s+2)+k-s] / 2} \lambda^{k / d}} \\
& \times \int_{0}^{\pi} \int_{0}^{\pi-\beta_{1}}\left(\sin \beta_{1} \sin \beta_{2}\right)^{d s+d-s-1} \sin ^{k}\left(\beta_{1}+\beta_{2}\right) \\
& \times\left(\sum_{i=0}^{\lfloor(d-1) / 2\rfloor} b_{i}\left(\beta_{1}\right)\right)\left(\sum_{i=0}^{\lfloor(d-1) / 2\rfloor} b_{i}\left(\beta_{2}\right)\right) \\
& \times\left\{\sin ^{d} \beta_{2} \sum_{i=0}^{\lfloor d / 2\rfloor} a_{i}\left(\beta_{1}\right)+\sin ^{d} \beta_{1} \sum_{i=0}^{\lfloor d / 2\rfloor} a_{i}\left(\beta_{2}\right)\right\}^{-[s+2+(k-s) / d]} \mathrm{d} \beta_{2} \mathrm{~d} \beta_{1}, \\
& k=0,1,2, \ldots, \quad
\end{aligned}
$$

replacing the three-fold integral by a two-fold one. The first moment of edge length can be given explicitly by the use of symmetry arguments. The mean of the projection of $z$ onto $E_{d, s}$ is its midpoint. Hence,

$\mathrm{E} L_{d, s}=2 \mathrm{E} \Delta_{d, s} \mathrm{E} \cos B_{d, s}$

$$
=\frac{\Gamma\left(\frac{1}{2}(d+1)\right) \Gamma\left(\frac{1}{2}(d s+d-s)\right) \Gamma\left(\frac{1}{2}(d s-s+2)\right) \Gamma(s-(s-1) / d)}{\left\{\Gamma\left(\frac{1}{2}(d+2)\right)\right\}^{1-1 / d} \Gamma\left(\frac{1}{2}(d s+d-s+1)\right) \Gamma\left(\frac{1}{2}(d s-s+1)\right) \Gamma(s+1-s / d) \lambda^{1 / d}} .
$$

This result was given in [11]. 


\section{Characteristics concerning neighbours}

The probability density function of the angle $A_{d, s}=\angle v_{1} z v_{2}$, at the neighbour $z$ of $E_{d, s}$, is $f_{A_{d, s}}(\alpha)$

$$
\begin{aligned}
& =\frac{2(d-1) s \Gamma\left(\frac{1}{2}(d+1)\right) \Gamma\left(\frac{1}{2}(d s+d-s+2)\right)}{\Gamma\left(\frac{1}{2} d\right) \Gamma\left(\frac{1}{2}(d s+d-s+1)\right)} \\
& \quad \times \int_{0}^{\pi-\alpha}\left(\sin \beta_{1} \sin \left(\alpha+\beta_{1}\right)\right)^{d s+d-s-1}\left(\sum_{i=0}^{\lfloor(d-1) / 2\rfloor} b_{i}\left(\beta_{1}\right)\right)\left(\sum_{i=0}^{\lfloor(d-1) / 2\rfloor} b_{i}\left(\pi-\alpha-\beta_{1}\right)\right) \\
& \quad \times\left\{\sin ^{d}\left(\alpha+\beta_{1}\right) \sum_{i=0}^{\lfloor d / 2\rfloor} a_{i}\left(\beta_{1}\right)+\sin ^{d} \beta_{1} \sum_{i=0}^{\lfloor d / 2\rfloor} a_{i}\left(\pi-\alpha-\beta_{1}\right)\right\}^{-[s+2-s / d]} \mathrm{d} \beta_{1}, \\
&
\end{aligned}
$$

This generalizes a result given in [1] (for $f_{A_{2,2}}(\alpha)$ ). The corresponding means are given by

$$
\mathrm{E} A_{d, s}=\pi-2 \mathrm{E} B_{d, s}=\frac{\sqrt{\pi} \Gamma\left(\frac{1}{2}(d+1)\right) \Gamma\left(\frac{1}{2}(d s+d-s)\right) \Gamma\left(\frac{1}{2}(d s-s+1)\right)}{\Gamma\left(\frac{1}{2} d\right) \Gamma\left(\frac{1}{2}(d s+d-s+1)\right) \Gamma\left(\frac{1}{2}(d s-s+2)\right)} .
$$

We call an edge incentric or excentric if the joint foot of the perpendiculars of its neighbours is inside or outside the edge, respectively. In the special case $d=2$, an incentric edge corresponds to a pair of direct neighbours and an excentric one to a pair of indirect neighbours. For $d \geq 2$, each excentric edge represents a pair of indirect neighbours, but each incentric edge does not necessarily represent a pair of direct neighbours. The probabilities of the typical edge $E_{d, s}$ to be incentric or excentric are respectively given by

$$
\begin{gathered}
p_{d, s, \text { inc }}=\frac{\Gamma\left(\frac{1}{2}(d+1)\right) \Gamma\left(\frac{1}{2}(d s+d-s)\right)}{\Gamma\left(\frac{1}{2} d\right) \Gamma\left(\frac{1}{2}(d s+d-s+1)\right)}, \\
p_{d, s, \text { exc }}=1-\frac{\Gamma\left(\frac{1}{2}(d+1)\right) \Gamma\left(\frac{1}{2}(d s+d-s)\right)}{\Gamma\left(\frac{1}{2} d\right) \Gamma\left(\frac{1}{2}(d s+d-s+1)\right)} .
\end{gathered}
$$

The probability density function of the length of the typical edge can be split into two parts, one for the typical incentric edge and one for the typical excentric edge, as follows:

$$
\begin{aligned}
& f_{L_{d, s, \text { inc }}}(l)=\frac{1}{p_{d, s, \text { inc }}} \int_{0}^{\pi / 2} \int_{0}^{\pi / 2} f_{\left\{L, B_{1}, B_{2}\right\}_{d, s}}\left(l, \beta_{1}, \beta_{2}\right) \mathrm{d} \beta_{2} \mathrm{~d} \beta_{1}, \\
& f_{L_{d, s, \text { exc }}}(l)=\frac{2}{p_{d, s, \text { exc }}} \int_{\pi / 2}^{\pi} \int_{0}^{\pi-\beta_{1}} f_{\left\{L, B_{1}, B_{2}\right\}_{d, s}}\left(l, \beta_{1}, \beta_{2}\right) \mathrm{d} \beta_{2} \mathrm{~d} \beta_{1} .
\end{aligned}
$$

The probability density function of the height $H_{d, s}$ over $E_{d, s}$ of the neighbour $z$ (the distance from $z$ to the foot of its perpendicular onto the typical edge or onto the straight line containing it) is given by

$$
\begin{aligned}
f_{H_{d, s}}(h)= & \frac{d(d-1) s \Gamma\left(\frac{1}{2}(d+1)\right) \Gamma\left(\frac{1}{2}(d s+d-s)\right)\left(\lambda \omega_{d}\right)^{s+1-s / d}}{\Gamma\left(\frac{1}{2} d\right) \Gamma\left(\frac{1}{2}(d s+d-s+1)\right) \Gamma(s+1-s / d)} \\
& \times \int_{0}^{\pi} \frac{h^{(d-1)(s+1)}}{\sin ^{d+1} \beta}\left\{\sum_{i=0}^{\lfloor(d-1) / 2\rfloor} b_{i}(\beta)\right\} \exp \left(-\frac{\lambda \omega_{d} h^{d}}{\sin ^{d} \beta}\right) \mathrm{d} \beta, \quad h \geq 0 .
\end{aligned}
$$


In the special case $d=2$, the function can be simplified to

$$
f_{H_{2, s}}(h)=\frac{s(\lambda \pi)^{s / 2}}{\Gamma\left(\frac{1}{2}(s+3)\right)}\left\{\sqrt{\lambda \pi} h^{s} \exp \left(-\lambda \pi h^{2}\right)+h^{s-1} \int_{h \sqrt{\lambda \pi}}^{\infty} \exp \left(-x^{2}\right) \mathrm{d} x\right\}, \quad h \geq 0 .
$$

This is a generalization of a result given by Brakke [1] and Collins [3], [4] for $d=s=2$. Brakke and Collins considered the distance between two neighbours positioned symmetrically on either side of the edge, which is twice $H_{2,2}$. Collins also indicated a formulation for $d=3$. The moments corresponding to (23) are

$$
\begin{aligned}
\mathrm{E} H_{d, s}^{k}=\frac{\Gamma\left(\frac{1}{2}(d s+d-s)\right) \Gamma\left(\frac{1}{2}(d s+d+k-s+1)\right) \Gamma(s+(k-s) / d)}{\Gamma\left(\frac{1}{2}(d s+d+k-s)\right) \Gamma\left(\frac{1}{2}(d s+d-s+1)\right) \Gamma(s-s / d)\left(\lambda \omega_{d}\right)^{k / d}}, \\
k=0,1,2, \ldots
\end{aligned}
$$

Finally, consider the position of the neighbour $z$ relative to $E_{d, s}$. We scale the space by a factor of $L_{d, s}$, so that the typical edge is just a segment of unit length with endpoints at $-\frac{1}{2}$ and $\frac{1}{2}$ on the $x_{1}$-axis. Denote by $X$ the random position of $z$ on the $x_{1}$-axis (the coordinate of its projection), and by $Y$ the distance of $z$ to the $x_{1}$-axis (the length of the perpendicular). Then the joint probability density function of the (scaled) location of $z=(X, Y)$ is

$$
\begin{aligned}
f_{\{X, Y\}_{d, s}(x, y)=} & \frac{2(d-1) s \Gamma\left(\frac{1}{2}(d+1)\right) \Gamma\left(\frac{1}{2}(d s+d-s+2)\right)}{\Gamma\left(\frac{1}{2} d\right) \Gamma\left(\frac{1}{2}(d s+d-s+1)\right)} \\
& \times y^{d s-s-1}\left\{\rho_{+} \rho_{-}\right\}^{d-1}\left(\sum_{i=0}^{\lfloor(d-1) / 2\rfloor} b_{i}\left(\frac{1}{2} \pi-\arctan \frac{\frac{1}{2}+x}{y}\right)\right) \\
& \times\left(\sum_{i=0}^{\lfloor(d-1) / 2\rfloor} b_{i}\left(\frac{1}{2} \pi-\arctan \frac{\frac{1}{2}-x}{y}\right)\right) \\
& \times\left\{\rho_{+}^{d} \sum_{i=0}^{\lfloor d / 2\rfloor} a_{i}\left(\frac{1}{2} \pi-\arctan \frac{\frac{1}{2}+x}{y}\right)\right. \\
& \left.\quad+\rho_{-}^{d} \sum_{i=0}^{\lfloor d / 2\rfloor} a_{i}\left(\frac{1}{2} \pi-\arctan \frac{\frac{1}{2}-x}{y}\right)\right\}^{-[s+2-s / d]},
\end{aligned}
$$

with $-\infty<x<\infty, 0 \leq y<\infty, \rho_{+}=\left[\left(\frac{1}{2}+x\right)^{2}+y^{2}\right]^{1 / 2}$, and $\rho_{-}=\left[\left(\frac{1}{2}-x\right)^{2}+y^{2}\right]^{1 / 2}$.

\section{Numerical calculations and special values}

The characteristics considered in this section were calculated by numerical evaluation of the integral formulae in Sections 3 and 4. The numerical computations were made partly by the use of MATHEMATICA ${ }^{\circledR}$ and partly by procedures written by the author. Probability density functions of the length $L_{d, s}$ of the typical edge and the angle $A_{d, s}$ spanned at one of its neighbours are given for the special cases $d=2$ and $d=3$, including all corresponding sections $(1 \leq s \leq d)$, and for $d=25$ with $s=1,13,25$; see Figures 1 and 2 and Tables 1 and 2. Furthermore, Table 2 gives the location $y^{*}$ of the maximum value $f_{\{X, Y\}_{d, s}}\left(0, y^{*}\right)$ of the probability density function of the position of the neighbours relative to the typical edge, when scaled to a segment of unit length. (Some other tables and figures of further characteristics investigated in this paper can be obtained from the author.) 


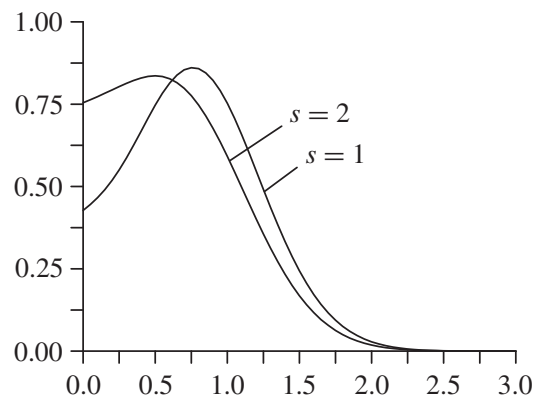

(a)

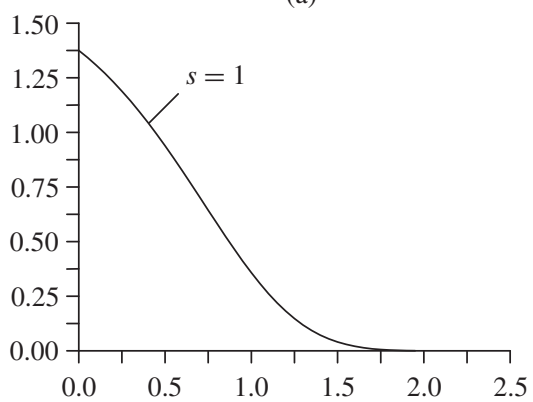

(c)

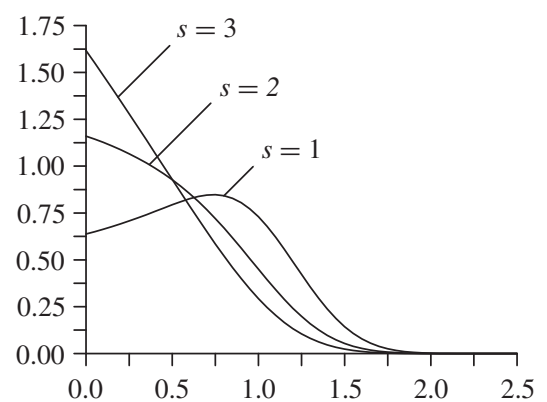

(b)

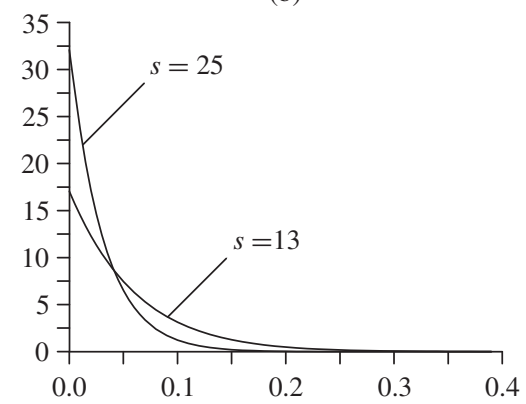

(d)

FIGURE 1: Probability density function of the length $L_{d, s}$ of the typical edge, plotted against $l$ for (a) $d=2$, (b) $d=3$ (this plot is given in [14]), (c) $d=25$, and (d) $d=25$.

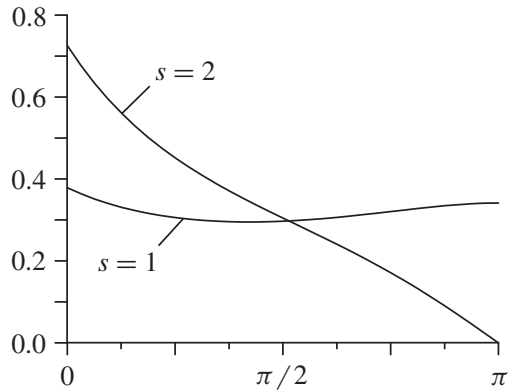

(a)

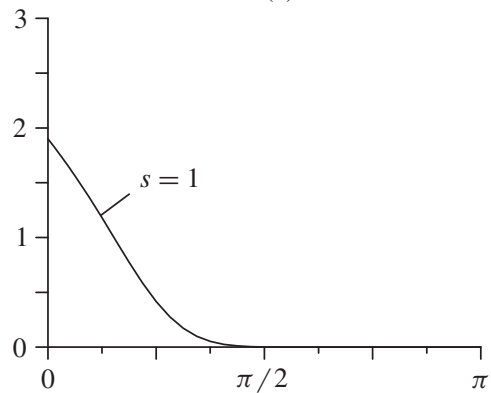

(c)

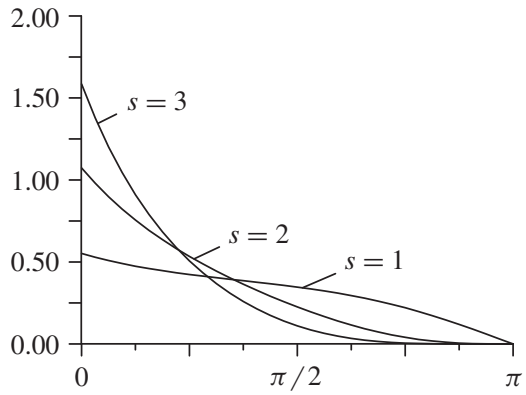

(b)

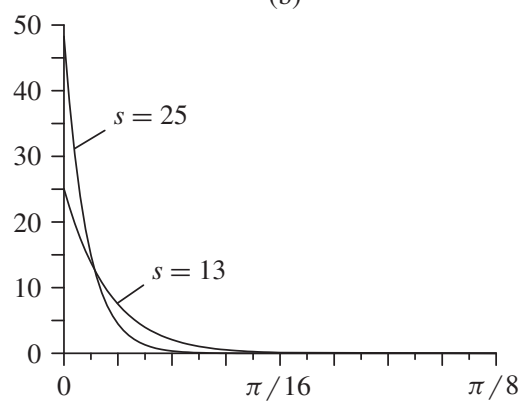

(d)

Figure 2: Probability density function of the angle $A_{d, s}$ spanned, at a neighbour of the typical edge, by its endpoints, plotted against $\alpha$ for (a) $d=2$, (b) $d=3$, (c) $d=25$, and (d) $d=25$. 
TABLE 1: Moments $\mathrm{E} L_{d, s}^{k}$ of the length $L_{d, s}$ of the typical edge.

\begin{tabular}{rrcccll}
\hline \multicolumn{1}{c}{$d$} & \multicolumn{1}{c}{$s$} & \multicolumn{1}{c}{$k=1$} & \multicolumn{1}{c}{$k=2$} & \multicolumn{1}{c}{$k=3$} & \multicolumn{1}{c}{$k=4$} & \multicolumn{1}{c}{$k=5$} \\
\hline 2 & 1 & 0.785398 & 0.806079 & 0.960132 & 1.27335 & 1.83990 \\
2 & 2 & 0.666667 & 0.630072 & 0.713921 & 0.915693 & 1.29194 \\
3 & 1 & 0.687182 & 0.631044 & 0.667220 & 0.773694 & 0.961232 \\
3 & 2 & 0.522612 & 0.403531 & 0.376644 & 0.396673 & 0.455961 \\
3 & 3 & 0.430858 & 0.290878 & 0.245190 & 0.238011 & 0.255703 \\
25 & 1 & 0.473580 & 0.343279 & 0.307221 & 0.314522 & 0.354664 \\
25 & 13 & 0.0559009 & $5.98 \times 10^{-3}$ & $9.21 \times 10^{-4}$ & $1.82 \times 10^{-4}$ & $4.33 \times 10^{-5}$ \\
25 & 25 & 0.0303855 & $1.80 \times 10^{-3}$ & $1.57 \times 10^{-4}$ & $1.78 \times 10^{-5}$ & $2.46 \times 10^{-6}$ \\
\hline
\end{tabular}

TABLE 2: Moments of the angle $A_{d, s}$ spanned, at a neighbour of the typical edge, by its endpoints, and moments of the corresponding cosine; the position $y^{*}$ of the maximum $f_{\{X, Y\}_{d, s}}\left(0, y^{*}\right)$; and the corresponding maximum value $f_{\{X, Y\}_{d, s}}\left(0, y^{*}\right)$ itself.

\begin{tabular}{rrrlllll}
\hline$d$ & $s$ & \multicolumn{1}{c}{$\mathrm{E} A_{d, s}$} & \multicolumn{1}{c}{$\mathrm{E} A_{d, s}^{2}$} & $\mathrm{E}\left(\cos A_{d, s}\right)$ & $\mathrm{E}\left(\cos A_{d, s}\right)^{3}$ & $y^{*}$ & $f_{\{X, Y\}_{d, s}}\left(0, y^{*}\right)$ \\
\hline 2 & 1 & 1.57080 & 3.33333 & $-1.71481 \times 10^{-3}$ & $1.81717 \times 10^{-3}$ & 0.0000 & 3.3322 \\
2 & 2 & 1.04720 & 1.67975 & 0.400882 & 0.320078 & 0.2534 & 1.4858 \\
3 & 1 & 1.17810 & 2.00254 & 0.299916 & 0.241124 & 0.2338 & 2.4960 \\
3 & 2 & 0.736311 & 0.874620 & 0.644065 & 0.503758 & 0.4410 & 1.1507 \\
3 & 3 & 0.536893 & 0.491878 & 0.785343 & 0.638107 & 0.6160 & 0.67102 \\
25 & 1 & 0.359921 & 0.202693 & 0.903379 & 0.775919 & 1.107 & 1.2619 \\
25 & 13 & 0.0382649 & $2.816 \times 10^{-3}$ & 0.998594 & 0.995812 & 8.764 & 0.016553 \\
25 & 25 & 0.0202626 & $8.038 \times 10^{-4}$ & 0.999599 & 0.998798 & 16.49 & 0.0059261 \\
\hline
\end{tabular}

In the following, values of probability density functions for particular arguments are given. First, we have

$$
f_{B_{d, s}}(0)= \begin{cases}\frac{1}{4} \pi, & d=2, s=1, \\ 0, & d+s>3\end{cases}
$$

The use of (5) for $l=0$ gives

$$
f_{L_{d, s}}(0)=\frac{d^{2}\left(\lambda \omega_{d}\right)^{1 / d} \Gamma\left(\frac{1}{2}(d+1)\right) \Gamma\left(\frac{1}{2}(d s+d-s)\right) \Gamma(s+2-(s+1) / d)}{\Gamma(s-s / d) \Gamma\left(\frac{1}{2} d\right) \Gamma\left(\frac{1}{2}(d s+d-s+1)\right)} \int_{0}^{\pi} w(\beta) \mathrm{d} \beta,
$$

where

$$
w(\beta)=\sin ^{d s-s-1} \beta\left(\sum_{i=0}^{\lfloor(d-1) / 2\rfloor} b_{i}(\beta)\right)\left(\sum_{i=0}^{\lfloor(d-1) / 2\rfloor} b_{i}(\pi-\beta)\right) .
$$

From

$$
\begin{aligned}
f_{L_{d, s, \mathrm{exc}}}(0)= & \frac{2 d^{2}\left(\lambda \omega_{d}\right)^{1 / d} \Gamma\left(\frac{1}{2}(d+1)\right) \Gamma\left(\frac{1}{2}(d s+d-s)\right) \Gamma(s+2-(s+1) / d)}{\Gamma(s-s / d)\left\{\Gamma\left(\frac{1}{2} d\right) \Gamma\left(\frac{1}{2}(d s+d-s+1)\right)-\Gamma\left(\frac{1}{2}(d+1)\right) \Gamma\left(\frac{1}{2}(d s+d-s)\right)\right\}} \\
& \times \int_{\pi / 2}^{\pi} w(\beta) \mathrm{d} \beta \\
f_{L_{d, s, \mathrm{inc}}}(0)= & 0,
\end{aligned}
$$


we see that only excentric edges contribute to the probability density function of the length of the typical edge at $l=0$. Finally, we have

$$
\begin{aligned}
& f_{A_{d, s}}(0)=\frac{2(d-1) s \Gamma\left(\frac{1}{2}(d+1)\right) \Gamma\left(\frac{1}{2}(d s+d-s+2)\right)}{\Gamma\left(\frac{1}{2} d\right) \Gamma\left(\frac{1}{2}(d s+d-s+1)\right)} \\
& \times \int_{0}^{\pi} \sin ^{d s-s-2} \beta_{1}\left(\sum_{i=0}^{\lfloor(d-1) / 2\rfloor} b_{i}\left(\beta_{1}\right)\right)\left(\sum_{i=0}^{\lfloor(d-1) / 2\rfloor} b_{i}\left(\pi-\beta_{1}\right)\right) \mathrm{d} \beta_{1}, \\
& f_{A_{d, s}}(\pi)= \begin{cases}\frac{\pi}{8}\left(\frac{3 \log (\sqrt{2}+1)}{\sqrt{2}}-1\right) \approx 0.341521, & d=2, s=1, \\
0, & d+s>3,\end{cases} \\
& f_{H_{d, s}}(0)= \begin{cases}\frac{1}{2} \pi \lambda^{1 / 2}, & d=2, s=1 \\
0, & d+s>3\end{cases} \\
& f_{\{X, Y\}_{d, s}}(0,0)= \begin{cases}\frac{3 \pi}{2 \sqrt{2}}, & d=2, s=1 \\
0, & d+s>3\end{cases}
\end{aligned}
$$

\section{Proofs}

Three basic integrals often used in the following are

$$
\begin{aligned}
\int_{0}^{\infty} t^{a} \exp \left(-b t^{c}\right) \mathrm{d} t & =\frac{1}{c} \Gamma\left(\frac{a+1}{c}\right)\left(\frac{1}{b}\right)^{(a+1) / c}, \\
\int_{0}^{\pi} \sin ^{a} t \mathrm{~d} t & =\sqrt{\pi} \frac{\Gamma\left(\frac{1}{2}(a+1)\right)}{\Gamma\left(\frac{1}{2}(a+2)\right)}, \\
\int_{0}^{\pi}(\pi-t) \sin ^{a} t \cos t \mathrm{~d} t & =\frac{1}{a+1} \int_{0}^{\pi} \sin ^{a+1} t \mathrm{~d} t,
\end{aligned}
$$

with $0<a, b, c \in \mathbb{R}$. Furthermore, summation formulae for $\Gamma$-terms are, for $d>2$,

$$
\left\{\begin{array}{l}
\sum_{i=1}^{d / 2-1} \frac{\Gamma(i) \Gamma\left(\frac{1}{2}(m+2 i+1)\right)}{\Gamma\left(i+\frac{3}{2}\right) \Gamma\left(\frac{1}{2}(m+2 i+2)\right)} \\
=\frac{8}{\sqrt{\pi} m} \frac{\Gamma\left(\frac{1}{2}(m+3)\right)}{\Gamma\left(\frac{1}{2}(m+2)\right)}-\frac{4}{m} \frac{\Gamma\left(\frac{1}{2} d\right)}{\Gamma\left(\frac{1}{2}(d+1)\right)} \frac{\Gamma\left(\frac{1}{2}(d+m+1)\right)}{\Gamma\left(\frac{1}{2}(d+m)\right)}, \quad d \text { even, } \\
\sum_{i=1}^{\frac{1}{2}(d-1)} \frac{\Gamma\left(i-\frac{1}{2}\right) \Gamma\left(\frac{1}{2}(m+2 i)\right)}{\Gamma(i+1) \Gamma\left(\frac{1}{2}(m+2 i+1)\right)} \\
=\frac{2 \sqrt{\pi} \Gamma\left(\frac{1}{2} m\right)}{\Gamma\left(\frac{1}{2}(m+1)\right)}-\frac{4}{m} \frac{\Gamma\left(\frac{1}{2} d\right)}{\Gamma\left(\frac{1}{2}(d+1)\right)} \frac{\Gamma\left(\frac{1}{2}(d+m+1)\right)}{\Gamma\left(\frac{1}{2}(d+m)\right)}, \quad d \text { odd },
\end{array}\right.
$$

for $m=1,2, \ldots$ 
Proof of (30). The equality can be shown immediately for $d=3$ and $d=4$ for each $m \geq 1$. To handle the case of even $d$ by induction, we write

$$
\begin{aligned}
K_{d, m} & =\sum_{i=1}^{d / 2-1} \frac{\Gamma(i)}{\Gamma\left(i+\frac{3}{2}\right)} \frac{\Gamma\left(\frac{1}{2}(m+2 i+1)\right)}{\Gamma\left(\frac{1}{2}(m+2 i+2)\right)} \\
& =\frac{8}{\sqrt{\pi} m} \frac{\Gamma\left(\frac{1}{2}(m+3)\right)}{\Gamma\left(\frac{1}{2}(m+2)\right)}-\frac{4}{m} \frac{\Gamma\left(\frac{1}{2} d\right)}{\Gamma\left(\frac{1}{2}(d+1)\right)} \frac{\Gamma\left(\frac{1}{2}(d+m+1)\right)}{\Gamma\left(\frac{1}{2}(d+m)\right)}
\end{aligned}
$$

the second equality holds for $d=4$. Then,

$$
\begin{aligned}
K_{d+2, m} & =K_{d, m}+\frac{\Gamma\left(\frac{1}{2} d\right)}{\Gamma\left(\frac{1}{2}(d+3)\right)} \frac{\Gamma\left(\frac{1}{2}(d+m+1)\right)}{\Gamma\left(\frac{1}{2}(d+m+2)\right)} \\
& =\frac{8}{\sqrt{\pi} m} \frac{\Gamma\left(\frac{1}{2}(m+3)\right)}{\Gamma\left(\frac{1}{2}(m+2)\right)}-\frac{4}{m} \frac{\Gamma\left(\frac{1}{2} d+1\right)}{\Gamma\left(\frac{1}{2}(d+3)\right)} \frac{\Gamma\left(\frac{1}{2}(d+m+3)\right)}{\Gamma\left(\frac{1}{2}(d+m+2)\right)}
\end{aligned}
$$

proves the first line of (30). The equality can be shown analogously for odd $d$.

Proof of (9). The $d$-volume of a truncated sphere of radius $r$ and intersection longitude $\beta$ is given, without loss of generality, by

$$
\begin{aligned}
\varpi_{d}(r, \beta) & =\overbrace{\left\{x_{i}: \sum_{i=1}^{d} \cdots \int\right.}^{d} \prod_{\left.x_{i}^{2} \leq r^{2}, x_{d} \leq r \cos \beta\right\}}^{d} \mathrm{~d} x_{i} \\
& =r^{d} \varpi_{d}(1, \beta),
\end{aligned}
$$

with Cartesian coordinates $x_{i}, i=1, \ldots, d$, which can be simplified by recursion to

$$
\begin{aligned}
\varpi_{d}(r, \beta) & =r^{d} \int_{-1}^{\cos \beta} \varpi_{d-1}\left(\sqrt{1-x_{d}^{2}}, 0\right) \mathrm{d} x_{d} \\
& =r^{d} \varpi_{d-1}(1,0) \int_{-1}^{\cos \beta}\left(1-x_{d}^{2}\right)^{(d-1) / 2} \mathrm{~d} x_{d} .
\end{aligned}
$$

Here, $\left(1-x_{d}^{2}\right)^{1 / 2}$ is the radius of the intersection between the complete sphere $b(o, 1)$ and the hyperplane $x=x_{d}$. For complete spheres, the relationship

$$
\varpi_{k}(1,0)=\varpi_{k-1}(1,0) \int_{0}^{\pi} \sin ^{k} \varphi \mathrm{d} \varphi, \quad k>1,
$$

holds. Repeated recursion and the substitution $x_{d}=\cos \varphi$ lead to

$$
\varpi_{d}(1, \beta)=\prod_{i=1}^{d-1}\left(\int_{0}^{\pi} \sin ^{i} \varphi \mathrm{d} \varphi\right) \int_{\beta}^{\pi} \sin ^{d} \varphi \mathrm{d} \varphi, \quad d \geq 2 .
$$


Integration by parts and recursion give

$$
\int_{\beta}^{\pi} \sin ^{n+2} \varphi \mathrm{d} \varphi=\frac{n+1}{n+2} \int_{\beta}^{\pi} \sin ^{n} \mathrm{~d} \varphi+\frac{1}{n+2} \sin ^{n+1} \beta \cos \beta, \quad n \geq 0,
$$

which leads to

$$
\begin{aligned}
\int_{\beta}^{\pi} \sin ^{d} \varphi \mathrm{d} \varphi \\
\quad= \begin{cases}\frac{\Gamma\left(\frac{1}{2}(d+1)\right)}{\Gamma\left(\frac{1}{2} d+1\right)}\left(\sqrt{\pi}\left(1-\frac{\beta}{\pi}\right)+\frac{\cos \beta}{2} \sum_{i=1}^{d / 2} \frac{\Gamma(i)}{\Gamma\left(i+\frac{1}{2}\right)} \sin ^{2 i-1} \beta\right), & d \text { even, } \\
\frac{\Gamma\left(\frac{1}{2}(d+1)\right)}{\Gamma\left(\frac{1}{2} d+1\right)}\left(\sqrt{\pi} \cos ^{2} \frac{1}{2} \beta+\frac{\cos \beta}{2} \sum_{i=1}^{(d-1) / 2} \frac{\Gamma\left(i+\frac{1}{2}\right)}{\Gamma(i+1)} \sin ^{2 i} \beta\right), & d \text { odd },\end{cases}
\end{aligned}
$$

and use of (28) gives (9) and (10). The union of two overlapping spheres with radii $l \sin \beta_{2} / \sin \left(\beta_{1}+\beta_{2}\right)$ and $l \sin \beta_{1} / \sin \left(\beta_{1}+\beta_{2}\right)$ and midpoint distance $l$ is the same as the union of two truncated spheres with intersection longitudes $\beta_{1}$ and $\beta_{2}$ and radii $l \sin \beta_{2} / \sin \left(\beta_{1}+\beta_{2}\right)$ and $l \sin \beta_{1} / \sin \left(\beta_{1}+\beta_{2}\right)$, respectively. This observation leads to (8).

Proof of (6). Recall that each vertex in the $s$-dimensional section $\mathfrak{V}_{s}$ is the intersection point with a $(d-s)$-facet of $\mathfrak{V}$ in $\mathbb{R}^{d}$. Consider the typical vertex $o$ of $\mathfrak{V}_{s}$, which has $s+1$ equidistant nearest neighbours at a distance $\delta$. The interior of the sphere $b(o, \delta)$ contains no neighbours, but its boundary $\partial b(o, \delta)$ contains $s+1$ neighbours. Following [8], the point configuration outside $b(o, \delta)$ can be handled in the same way as can a Poisson point process. The statement given in [8, Lemmas 1 and $1^{\prime}$ ] can be generalized to section hyperplanes of each dimension $s=$ $1,2, \ldots, d$. The region in $b(o, \delta)$ can be considered to be a Poisson point process conditioned on the event that there are $s+1$ points on the boundary $\partial b(o, \delta)$ and none in its interior. To determine the distribution function $F_{\Delta_{d, s}}$ of the distance $\Delta_{d, s}$ of the nearest neighbours to the typical vertex, it can be imagined that these $s+1$ neighbours are located in a thin hull $b(o, \delta+\varepsilon) \backslash b^{\text {int }}(o, \delta)$. (The probability of the event that there are more than $s+1$ points in this hull is neglected.) Then $F_{\Delta_{d, s}}$ can be written as a conditional probability in the following way:

$$
F_{\Delta_{d, s}}(\delta)=\lim _{\varepsilon \downarrow 0} \frac{\int_{0}^{\delta} \mathrm{P}\left(\Phi\left(b^{\text {int }}(o, x)\right)=0, \Phi(b(o, x+\varepsilon))=s+1\right) \mathrm{d} x}{\int_{0}^{\infty} \mathrm{P}\left(\Phi\left(b^{\text {int }}(o, x)\right)=0, \Phi(b(o, x+\varepsilon))=s+1\right) \mathrm{d} x} .
$$

The use of well-known distributional properties of Poisson point patterns gives

$$
F_{\Delta_{d, s}}(\delta)=\lim _{\varepsilon \downarrow 0} \frac{\int_{0}^{\delta}\left(\lambda \omega_{d}\right)^{s+1} /(s+1) !\left\{(x+\varepsilon)^{d}-x^{d}\right\}^{s+1} \exp \left(-\lambda \omega_{d}(x+\varepsilon)^{d}\right) \mathrm{d} x}{\int_{0}^{\infty}\left(\lambda \omega_{d}\right)^{s+1} /(s+1) !\left\{(x+\varepsilon)^{d}-x^{d}\right\}^{s+1} \exp \left(-\lambda \omega_{d}(x+\varepsilon)^{d}\right) \mathrm{d} x} .
$$

Neglect of all $O\left(\varepsilon^{2}\right)$ terms in the integrand, and differentiation, lead to (6), while use of (27) yields (7).

Proof of (11). The derivative $v_{d}^{\prime}\left(l, \beta_{1}, \beta_{2}\right)$ is the volume of the difference set

$$
b\left(\underline{l+\mathrm{d} l},\left\|z_{0}-(l+\mathrm{d} l)\right\|\right) \backslash b\left(\underline{l},\left\|z_{0}-l\right\|\right)
$$


divided by $\mathrm{d} l$ or, equivalently, the derivative $\mathrm{d} v_{d}\left(l, \beta_{1}, \beta_{2}(l, \delta, \beta)\right) / \mathrm{d} l$ in (1) transformed according to (4). For a generic function $f$, let $f^{\prime}$ denote the derivative $\mathrm{d} f\left(l, \beta_{1}, \beta_{2}(l, \delta, \beta)\right) / \mathrm{d} l$ transformed according to (4). Then we obtain the following relationships:

$$
\begin{aligned}
\left(\left(\frac{l \sin \beta_{1}}{\sin \left(\beta_{1}+\beta_{2}\right)}\right)^{d}\right)^{\prime}= & d\left(\frac{l \sin \beta_{1}}{\sin \left(\beta_{1}+\beta_{2}\right)}\right)^{d-1} \cos \beta_{2}, \\
\left(\left(\frac{l \sin \beta_{1}}{\sin \left(\beta_{1}+\beta_{2}\right)}\right)^{d}\left(\pi-\beta_{2}\right)\right)^{\prime}= & \left(\frac{l \sin \beta_{1}}{\sin \left(\beta_{1}+\beta_{2}\right)}\right)^{d-1}\left(d\left(\pi-\beta_{2}\right) \cos \beta_{2}+\sin \beta_{2}\right), \\
\left(\left(\frac{l \sin \beta_{1}}{\sin \left(\beta_{1}+\beta_{2}\right)}\right)^{d} \cos \beta_{2} \sin ^{n} \beta_{2}\right)^{\prime}= & \left(\frac{l \sin \beta_{1}}{\sin \left(\beta_{1}+\beta_{2}\right)}\right)^{d-1} \\
& \times \sin ^{n} \beta_{2}\left(d-n-(d-n-1) \sin ^{2} \beta_{2}\right), \quad n \geq 0,
\end{aligned}
$$

and

$$
\begin{aligned}
\left(\left(\frac{l \sin \beta_{2}}{\sin \left(\beta_{1}+\beta_{2}\right)}\right)^{d}\right)^{\prime} & =\left(\left(\frac{l \sin \beta_{2}}{\sin \left(\beta_{1}+\beta_{2}\right)}\right)^{d}\left(\pi-\beta_{1}\right)\right)^{\prime} \\
& =\left(\left(\frac{l \sin \beta_{2}}{\sin \left(\beta_{1}+\beta_{2}\right)}\right)^{d} \cos \beta_{1} \sin ^{n} \beta_{1}\right)^{\prime}=0 .
\end{aligned}
$$

For even $d$, these expressions lead to

$$
\begin{aligned}
& v_{d}^{\prime}\left(l, \beta_{1}, \beta_{2}\right) \\
& =\omega_{d}\left(\frac{l \sin \beta_{1}}{\sin \left(\beta_{1}+\beta_{2}\right)}\right)^{d-1}\left\{\frac{d\left(\pi-\beta_{2}\right) \cos \beta_{2}+\sin \beta_{2}}{\pi}\right. \\
& \left.\quad+\frac{1}{2 \sqrt{\pi}} \sum_{i=1}^{d / 2} \frac{\Gamma(i)}{\Gamma\left(i+\frac{1}{2}\right)}\left((d-2 i+1) \sin ^{2 i-1} \beta_{2}-(d-2 i) \sin ^{2 i+1} \beta_{2}\right)\right\} \\
& =\omega_{d}\left(\frac{l \sin \beta_{1}}{\sin \left(\beta_{1}+\beta_{2}\right)}\right)^{d-1}\left\{\frac{d\left(\pi-\beta_{2}\right) \cos \beta_{2}+\sin \beta_{2}+(d-1) \sin \beta_{2}}{\pi}\right. \\
& \left.\quad+\frac{1}{2 \sqrt{\pi}} \sum_{i=1}^{d / 2-1}\left(\left\{-\frac{\Gamma(i)}{\Gamma\left(i+\frac{1}{2}\right)}(d-2 i)+\frac{\Gamma(i+1)}{\Gamma\left(i+\frac{3}{2}\right)}(d-2 i-1)\right\} \sin ^{2 i+1} \beta_{2}\right)\right\}
\end{aligned}
$$

and, thus, the first line of (12) is shown. The proof for odd $d$ is analogous.

Proof of (13). Inserting (11), (6), and (4) into the integrand of (3) and using the symmetric property $f_{L, B_{1}, B_{2}}\left(l, \beta_{1}, \beta_{2}\right)=f_{L, B_{1}, B_{2}}\left(l, \beta_{2}, \beta_{1}\right)$ gives

$$
f_{B_{d, s}}(\beta) \sim \sin ^{d s-s-1} \beta \sum_{i=0}^{\lfloor(d-1) / 2\rfloor} b_{i}(\beta), \quad 0 \leq \beta<\pi .
$$

To find the correct normalization, consider the integral expression

$$
I_{d, s}=\int_{0}^{\pi} \sin ^{d s-s-1} \beta \sum_{i=0}^{\lfloor(d-1) / 2\rfloor} b_{i}(\beta) \mathrm{d} \beta .
$$


The use of (28) and (29) gives, for $d>2$,

$$
I_{d, s}=\left\{\begin{array}{l}
\frac{d s-s+1}{\sqrt{\pi}(d-1) s} \frac{\Gamma\left(\frac{1}{2}(d s-s+1)\right)}{\Gamma\left(\frac{1}{2}(d s-s+2)\right)} \\
-\frac{1}{4} \sum_{i=1}^{d / 2-1} \frac{\Gamma(i)}{\Gamma\left(i+\frac{3}{2}\right)} \frac{\Gamma\left(\frac{1}{2}(d s-s+2 i+1)\right)}{\Gamma\left(\frac{1}{2}(d s-s+2 i+2)\right)}, \quad d \text { even, } \\
\frac{\sqrt{\pi}}{2} \frac{\Gamma\left(\frac{1}{2}(d s-s)\right)}{\Gamma\left(\frac{1}{2}(d s-s+1)\right)} \\
-\frac{1}{4} \sum_{i=1}^{(d-1) / 2} \frac{\Gamma\left(i-\frac{1}{2}\right)}{\Gamma(i+1)} \frac{\Gamma\left(\frac{1}{2}(d s-s+2 i)\right)}{\Gamma\left(\frac{1}{2}(d s-s+2 i+1)\right)}, \quad d \text { odd, }
\end{array}\right.
$$

and simplification using (30) leads to the normalization factor given in (13). The use of (29) and $\int_{0}^{\pi} \beta \sin ^{n} \beta \mathrm{d} \beta=\frac{1}{2} \pi \int_{0}^{\pi} \sin ^{n} \beta \mathrm{d} \beta$ gives (14) and (16) for odd $k$, while the use of (30) leads to (15).

Proof of (17), (18), (19), and (20). The use of (6), (13), (8), (9), (10), (11), (12), and (4) in (1) leads to (17), (18), and (19), while the use of (27) yields (20).

Proof of (21). The joint density of the neighbour-vertex-edge angles, obtained from (17) and (27), is

$$
\begin{aligned}
& f_{\left\{B_{1}, B_{2}\right\}_{d, s}\left(\beta_{1}, \beta_{2}\right)=} \frac{2(d-1) s \Gamma\left(\frac{1}{2}(d+1)\right) \Gamma\left(\frac{1}{2}(d s+d-s+2)\right)}{\Gamma\left(\frac{1}{2} d\right) \Gamma\left(\frac{1}{2}(d s+d-s+1)\right)} \\
& \times\left(\sin \beta_{1} \sin \beta_{2}\right)^{d s+d-s-1}\left(\sum_{i=0}^{\lfloor(d-1) / 2\rfloor} b_{i}\left(\beta_{1}\right)\right)\left(\sum_{i=0}^{\lfloor(d-1) / 2\rfloor} b_{i}\left(\beta_{2}\right)\right) \\
& \times\left\{\sin ^{d} \beta_{2} \sum_{i=0}^{\lfloor d / 2\rfloor} a_{i}\left(\beta_{1}\right)+\sin ^{d} \beta_{1} \sum_{i=0}^{\lfloor d / 2\rfloor} a_{i}\left(\beta_{2}\right)\right\}^{-[s+2-s / d]}, \\
& 0 \leq \beta_{2}<\pi-\beta_{1}, 0 \leq \beta_{1}<\pi .
\end{aligned}
$$

This formula generalizes results given in [13] for $f_{\left\{B_{1}, B_{2}\right\}_{d, s}}\left(\beta_{1}, \beta_{2}\right)$ with $2 \leq d \leq 3,2 \leq s \leq d$. An expression equivalent to (31) is given in [18]. Using the substitution $\beta_{2}=\pi-\alpha-\beta_{1}$ and integrating with respect to $\beta_{1}$ yields (21).

Proof of (22). The probability that the typical edge $E_{d, s}$ is incentric is

$$
p_{d, s, \text { inc }}=\mathrm{P}\left(B_{1_{d, s}}, B_{2_{d, s}} \leq \frac{1}{2} \pi\right) .
$$

From the symmetry of (31), the probability that the typical edge $E_{d, s}$ is excentric can be written as

$$
\begin{aligned}
p_{d, s, \text { exc }} & =\mathrm{P}\left(\text { either } B_{1_{d, s}} \text { or } B_{2_{d, s}} \text { is greater than } \frac{1}{2} \pi\right) \\
& =2 \int_{\pi / 2}^{\pi} \int_{0}^{\pi-\beta_{1}} f_{\left\{B_{1}, B_{2}\right\}_{d, s}}\left(\beta_{1}, \beta_{2}\right) \mathrm{d} \beta_{2} \mathrm{~d} \beta_{1}=2 \int_{\pi / 2}^{\pi} f_{B_{d, s}}(\beta) \mathrm{d} \beta .
\end{aligned}
$$

The use of (13), (28), (29), and (30) gives (22). 
Proof of (23). The distance $H_{d, s}$ from $z$ to the foot of its perpendicular on $E_{d, s}$, or simply the height of $z$, is equivalent to the product of $\Delta_{d, s}$ and $\sin B_{d, s}$. The distribution function can be written as

$$
F_{H_{d, s}}(h)=\underbrace{\int_{0}^{\infty} \int_{0}^{\pi}}_{H_{d, s}<h} f_{\Delta_{d, s}}(\delta) f_{B_{d, s}}(\beta) \mathrm{d} \delta \mathrm{d} \beta .
$$

Using the substitution $\delta=h / \sin \beta$ and differentiating with respect to $h$ leads to

$$
f_{H_{d, s}}(h)=\int_{0}^{\pi} f_{\Delta_{d, s}}\left(\frac{h}{\sin \beta}\right) f_{B_{d, s}}(\beta) \frac{1}{\sin \beta} \mathrm{d} \beta, \quad h \geq 0,
$$

and, thus, (23). The moments (25) are obtained from $\mathrm{E} H_{d, s}^{k}=\mathrm{E} \Delta_{d, s}^{k} \mathrm{E} \sin ^{k} B_{d, s}$, using (7) and (15).

To simplify (23) for the special case $d=2$, we write

$$
J_{\rho}(h)=h^{s} \exp \left(-\rho h^{2}\right) \quad \text { and } \quad J_{\tau}(h)=h^{s-1} \int_{\tau h}^{\infty} \exp \left(-x^{2}\right) \mathrm{d} x .
$$

Then, evaluation of the expression

$$
\begin{aligned}
r \int_{0}^{\infty} & h^{k} J_{\rho}(h) \mathrm{d} h+t \int_{0}^{\infty} h^{k} J_{\tau}(h) \mathrm{d} h \\
& =\frac{r}{2}\left(\frac{1}{\rho}\right)^{(k+s+1) / 2} \Gamma\left(\frac{1}{2}(k+s+1)\right)+\frac{t}{2(k+s)}\left(\frac{1}{\tau}\right)^{k+s} \Gamma\left(\frac{1}{2}(k+s+1)\right) \\
& =\mathrm{E} H_{2, s}^{k}, \quad k=0,1,2, \ldots,
\end{aligned}
$$

using (27) and (25), allows the determination of $r, \rho, t$, and $\tau$ via comparison of coefficients, and leads to (24).

Proof of (26). Finally, applying the transformation

$$
\begin{gathered}
\beta_{1}=\frac{1}{2} \pi-\arctan \frac{\frac{1}{2}+x}{y}, \quad \beta_{2}=\frac{1}{2} \pi-\arctan \frac{\frac{1}{2}-x}{y}, \\
\left|\frac{\partial\left(\beta_{1}, \beta_{2}\right)}{\partial(x, y)}\right|=\frac{y}{\left(\left(\frac{1}{2}+x\right)^{2}+y^{2}\right)\left(\left(\frac{1}{2}-x\right)^{2}+y^{2}\right)}
\end{gathered}
$$

in (31) leads to (26).

\section{Acknowledgements}

The author thanks Professor D. Stoyan for encouragement and for some critical remarks about, and helpful discussion of, an earlier version of this paper. The author also thanks the anonymous referee for some valuable comments and suggestions.

\section{References}

[1] Brakke, K. A. (1985). Statistics of random plane Voronoi tessellations. Preprint, Department of Mathematical Sciences, Susquehanna University Selinsgrove, PA.

[2] Brakke, K. A. (1985). Statistics of three dimensional random Voronoi tessellations. Preprint, Department of Mathematical Sciences, Susquehanna University Selinsgrove, PA. 
[3] Collins, R. (1968). A geometric sum rule for two-dimensional fluid correlation functions. J. Phys. C 1, 14611471.

[4] Collins, R. (1972). Melting and statistical geometry of simple liquids. In Phase Transitions and Critical Phenomena, eds C. Domb and M. S. Green, Academic Press, New York, pp. 271-303.

[5] Cowan, R., Quine, M. and Zuyev, S. (2003). Decomposition of gamma-distributed domains constructed from Poisson point processes. Adv. Appl. Prob. 35, 56-69.

[6] Li, X.-Y., WAN, P.-J. AND FrIeder, O. (2003). Coverage in wireless ad-hoc sensor networks. IEEE Trans. Comput. 526, 753-763.

[7] Liang, J. et al. (1998). Analytical shape computation of macromolecules. I. Molecular area and volume through alpha shape. Proteins Structure Function Genet. 33, 1-17.

[8] Mecke, J. And Muche, L. (1995). The Poisson Voronoi tessellation. I. A basic identity. Math. Nachr. 176, 199-208.

[9] Miles, R. E. (1970). On the homogeneous planar Poisson point process. Math. Biosci. 6, 85-127.

[10] Miles, R. E. (1974). A synopsis of 'Poisson flats in Euclidean spaces'. In Stochastic Geometry, eds E. F. Harding and D. G. Kendall, John Wiley, New York, pp. 202-227.

[11] Møller, J. (1989). Random tessellations in $\mathbb{R}^{d}$. Adv. Appl. Prob. 21, 37-73.

[12] MøLler, J. (1994). Lectures on Random Voronoi Tessellations (Lecture Notes Statist. 87). Springer, New York.

[13] Muche, L. (1996). The Poisson-Voronoi tessellation. II. Edge length distribution functions. Math. Nachr. 178, 271-283.

[14] Muche, L. (1999). Delaunay and Voronoi tessellation: Minkowski functionals and edge characteristics. Proc. $S^{4} G$ (Internat. Conf. Stereology, Spatial Statist. Stoch. Geom.; Prague, June 1999), eds V. Beneš, J. Janáček and I. Saxl, Union of Czech Mathematicians and Physicists, Prague, pp. 21-30.

[15] Muche, L. and Stoyan, D. (1992). Contact and chord length distributions of the Poisson Voronoi tessellation. J. Appl. Prob. 29, 467-471. (Correction: 30 (1993), 749.)

[16] Okabe, A., Boots, B., Sugihara, K. and Chiu, S. N. (2000). Spatial Tessellations: Concepts and Applications of Voronoi Diagrams, 2nd edn. John Wiley, Chichester.

[17] Sastry, S. et al. (1998). Free volume in the hard sphere liquid. Molec. Phys. 952, 289-297.

[18] Schlather, M. (2000). A formula for the edge length distribution function of the Poisson Voronoi tessellation. Math. Nachr. 214, 113-119.

[19] Stoyan, D., Kendall, W. S. And Mecke, J. (1995). Stochastic Geometry and Its Applications, 2nd edn. John Wiley, Chichester. 